\title{
Performance Comparison of Protocols Combination based on EIGRP and OSPF for Real-Time Applications in Enterprise Networks
}

\author{
Dounia EL IDRISSI \\ STIC Laboratory \\ Chouaib Doukkali University \\ El Jadida, Morocco \\ Najib ELKAMOUN \\ STIC Laboratory \\ Chouaib Doukkali University \\ El Jadida, Morocco
}

\author{
Fatima LAKRAMI \\ STIC Laboratory \\ Chouaib Doukkali University \\ El Jadida, Morocco \\ Rachid HILAL \\ STIC Laboratory \\ Chouaib Doukkali University \\ El Jadida, Morocco
}

\begin{abstract}
This work studies the impact of redistribution on network performance compared with the use of a single routing protocol. A real network with real traffic parameters is simulated, in order to investigate a real deployment case, and then being able to extract precise results and practical conclusions. This work demonstrates that using one single routing protocol is more efficient in general cases for real topologies, especially when deploying sensitive applications requiring a certain $Q 0 S$ level.
\end{abstract}

Keywords-Routing protocols; EIGRP; OSPF; Redistribution; QoS; Opnet

\section{INTRODUCTION}

Routing is the cement to ensure the cohesion of the Internet. Without it, TCP/IP traffic would be limited to a single physical network. Routing is the way to determine the optimal path of data between the transmitter and receiver. The routing is based on an algorithm that is specific to the routing protocol [1]. The algorithm takes into account the most important factors, such as average transmission time, network load, total message length, etc. It allows traffic from a local network to reach its destination wherever it is found in the world, after having crossed several intermediate networks. Routing is a task performed in many networks, such as the telephony network, electronic data networks (such as the internet, and transport networks). Its performance is important in decentralised networks, where information is not distributed by a single source but exchanged between independent agents.

The decisive role of routing and the complex interconnection of internet networks make the design of routing protocols a major challenge for network software developers. As a result, most routing studies involve protocol design; very few deal with the proper configuration of routing protocols. However, many day-to-day problems result rather from poor configuration of routers used than from the use of poorly designed algorithms. It is the role of the system administrator to ensure that the routing configuration is correct [2].
All routing protocols perform the same basic functions. They determine the best route to each destination and distribute routing information between systems in a network. The arrangements for carrying out these functions, in particular the procedures for selecting the best routes make it possible to distinguish between the various protocols [3]. But what happened exactly when the same network domain has to deal with different routing protocols? This is when the redistribution intervenes.

This paper investigates the impact of redistribution on network performance. In fact, the debate on the contribution of redistribution of routing protocols in the improvement/degradation of network performance is still an active research area, and for different schemes, different results can be obtained.

The main goal of this paper is to study, through experiment, the evolution of network performance while deploying the redistribution that consists of using more than one single routing protocol in one homogenous network. The study proposed here is based on a real fusion of two networks belonging to one enterprise, and communicating through an operator network.

The rest of the paper is organised as: Section 1 contains an introduction of routing problematic in large networks with real time applications. Section 2 contains a brief description of the routing protocols evaluated in this paper and the principle of the redistribution, Section 3 gives an idea about network planning and routing method choice, Section 4 contains related works, Section 5 presents problematic and contribution, Section 6 resumes simulation and results, and Section 7 concludes the paper.

\section{Routing PROTOCOLS AND REDISTRIBUTION}

The routing algorithm used to calculate route, and the metrics qualifying the best route to privilege a path among others, distinguish many routing protocols. There are various numbers of static and dynamic routing protocols available but 
the selection of appropriate routing protocol is most important for routing performance. The right choice of routing protocol is dependent on several parameters, related to network specifications and application requirements.

Actually, the Enhanced Interior Gateway Routing Protocol (EIGRP) and Open Shortest Path First (OSPF) are considered as the pre-eminent routing protocols for real-time applications. EIGRP is a Cisco proprietary distance-vector protocol based on Diffusing Update Algorithm (DUAL). On the other hand, OSPF is a link-state interior gateway protocol based on Dijkstra algorithm (Shortest Path First Algorithm).

EIGRP and OSPF are dynamic routing protocols used in practical networks to disseminate network topology to the adjacent routers. This work is based on the evaluation of combinations involving EIGRP and OSPF. A number of simulations have been done in order to compare different routing protocols. The obtained results showed that EIGRP and OSPF can be qualified as "better" routing protocols comparing with others.

\section{A. EIGRP}

Enhanced Interior Gateway Routing Protocol (EIGRP) is a routing protocol developed by Cisco based on their original IGRP protocol. EIGRP is an IP distance routing protocol with optimisation to minimise routing instability due to the topology changes, bandwidth utilisation, and router processor power. EIGRP uses a hybrid routing that relies on distance and link state vectors. The metrics used by EIGRP are thus mainly the bandwidth, the memory as well as the overhead of the processors. The EIGRP works quite differently from the IGRP. The EIGRP is an advanced distance vector routing protocol that acts as a link state protocol when updating neighbours and managing routing information [4]. Compared to simple distance vector protocols, it offers a number of advantages, especially for a rapid convergence time.

\section{B. $O S P F$}

OSPF (Open Shortest Path First) is a link state routing protocol that is used to distribute information within a single Autonomous System [5].

Its principle is that each router determines the state of its connections (links) with the neighbouring routers; it diffuses its information to all the routers belonging to the same zone. This information forms a database, which must be identical to all routers in the same zone. Knowing that a stand-alone system (AS) consists of several zones, all of these databases represent the topology of the AS.

\section{Redistribution of routing protocol}

Redistribution of routing protocols is defined as the use of a routing protocol to advertise roads that are learned by some other ways, such as by another routing protocol, static configuration, or directly connected roads. In fact, sometimes the use of multiprotocol routing becomes a necessity for a number of reasons, such as for company mergers, different services controlled by multiple network administrators, and multi-vendor environments. Running different routing protocols is often a part of designing a network. In any case, having a multi-protocol environment makes redistribution a necessity.

Differences in the characteristics of the routing protocol, such as metrics, administrative distance, class capabilities and classless can effect redistribution. Attention must be paid to these differences for redistribution to be a success. The principle of route redistribution consists in collecting the information relating to the routes learned via a routing protocol and injecting them into another routing domain. When a company or a community has several remote sites or nomadic users, they must be connected to communicate for exchanging data, applications, voice (IP telephony), etc. In particular, it allows companies with remote sites to benefit from access to their network wherever its geographic localisation remain.

Unfortunately, the redistribution of roads leads to several problems such as loss of metric, loss of administrative distance, redistribution loop and many others. This seems very logical. It is about two different algorithms unable to establish direct communication since they are speaking two different languages. The redistribution enables, in a certain manner, two different routing algorithms to exchange their routing data in order to cover the whole network. Despite this, redistribution still presents a number of problems. In fact, it can be described as a translator speaking a different language, destined to make two different routing protocols communicate, so it can be impossible for it to make a $100 \%$ correct translation in real time. This is the case of redistribution.

\section{NeTwork Planning AND Routing CHOICE}

From the point of view of enterprises, and in terms of data networks, the notion of sharing is simple: It is about optimising the use of resources by dividing them among different users. The optimisation referred to here is understood in at least two aspects: (1) the technical criterion, and (2) the economic criterion. This is referred to as technico-economic performance (or relevance). From the point of view of the operator, network conception must take into account the economic profitability, simplified changes and implementation deadlines.

Actually, enterprises make use of an operator connection to access their distributed resources, without having an idea about its network background or the transmission technology used in the backbone of the operator. Enterprises are now more aware about the necessity to cohabite with the operator in order to make benefit of a high level of quality of service to serve better their sensitive data. In fact, this is due to the evolution of the information systems of the most of the enterprises independently of their productive activities.

For this purpose, enterprises start to think about a way to communicate better with the operator, and then their branches. Among the choices that are available, there is the routing schema that must be adopted in order to accelerate or at least reduce latency and failures between their edge routers and the access router of the operator. This is why, practical studies must be done to evaluate and handle such connections or migrations. 


\section{RELATED WORKS}

Several works have been done to study the impact of routing protocols on the quality of the transmission of sensitive applications. In [6-9], authors prove by simulation of a communication using voice traffic that EIGRP shows better performance, especially for bandwidth management, rapid failure detection and for other performance metrics.

In [10], different combinations of multiple routing protocols have been configured (RIP_EIGRP, EIGRP_OSPF, OSPF_ISIS); they concluded that the best combination involves EIGRP and OSPF protocols. Authors also concluded that combining EIGRP and RIPv2 is better suited for small networks due to the absence of segmented areas. IS-IS is known as the most recommended protocol for ISP's and large enterprises because of its scalability and fast convergence. However, combining IS-IS with OSPF, shows better performance than configuring only one of them for any given scenario with complex parameters, due to their similarities. In [11], a detailed simulation analysis of the robustness of using OSPF, EIGRP and IS-IS together (OSPF/IS-IS and EIGRP/ISIS) compared with being deployed separately is given. Better performance is noticed when combining EIGRP and IS-IS.

Authors in [12], provide a comparative analysis of different routing protocols and their combination: EIGRP/OSPF, EIGRP/IS-IS, OSPF/IS-IS and EIGRP/IS-IS/OSPF. The study considered the case with real-time applications. Results obtained from simulations, show that the scenario with OSPF/IS-IS has manifest a minimal convergence time while scenario implementing the three protocols: (1) EIGRP, (2) ISIS, and (3) OSPF shows better performance for different metrics; Packet delay variation, packet End-to-End delay, Voice Jitter and link throughput. Therefore, authors conclude that the combination of those three protocols is more suitable, for the considered simulation.

In [13], a comparative analysis of some routing protocols such as EIGRP, OSPF and their combination has been evaluated in the same network for real time applications. Unlike previous cited works, [13] shows that the implementation of EIGRP, enable better convergence time, high throughput and less packet rate loss than using OSPF alone or combined with EIGRP.

The majority of cited works demonstrate the efficiency of the deployment of the redistribution. However, theatrically and practically it is not always the case, especially, when we cannot control the architecture of network topology [13], like the case covered in this work, where an examination of redistribution in a real case is proposed. The main goal is to prove its limitation, and its poor performance compared to using one single routing protocol.

\section{PROBLEMATIC AND CONTRIBUTION}

This paper is focused on the study of the case of an enterprise called "STIC" localised at Casablanca that just bought lately a new department localised at Tangier. STIC is willing to merge its two sites in order to make them communicate through a network connection provided by the operator.

The problem encountered here is the routing schema to be adopted, to make the two sites exchanging data, with real time traffic, in better conditions. So, the question was about which routing scheme is more suitable to provide an acceptable level of QoS? This work proposes to study, the better way, to connect at the IP level, the three networks that are presented successively by the site 1 of STIC, the operator backbone and the new site 2 of STIC (Figure 1). The study suggests different assumptions about routing plan for the operator side.

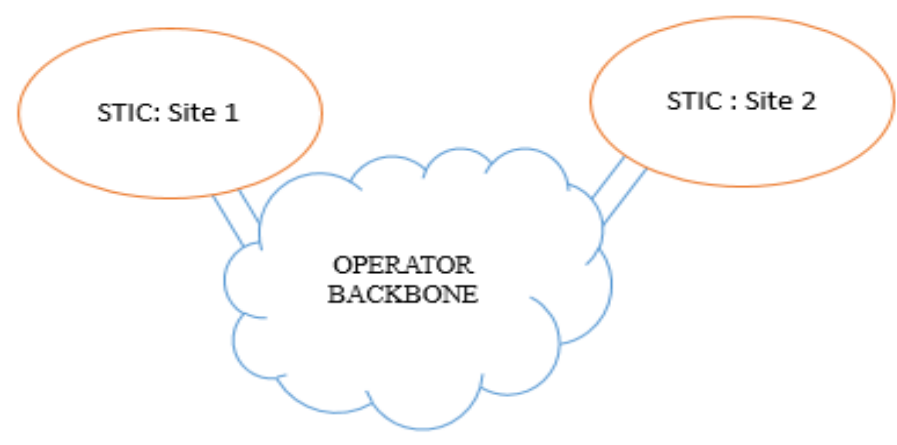

Fig. 1. The studied network topology

\section{Simulation AND RESUlts}

For the evaluation of the simulated topology, OPNET modeller 14.5 (Optimised Network Engineering Tools) has been used as a simulation environment.

OPNET is a high-level user interface that is built as of $\mathrm{C}$ and $\mathrm{C}++$ source code with huge library of OPNET function [14].

It is built on top of discrete event system (DES) and it simulates the system behaviour by modelling each event in the system and processes it through user defined processes. OPNET is very powerful software to simulate heterogeneous network with various protocols.

\section{A. Network Topology}

Figure 2 presents network topology, it is composed from two sites containing routers belonging to the STIC enterprise, and the operator network based on [15]. 


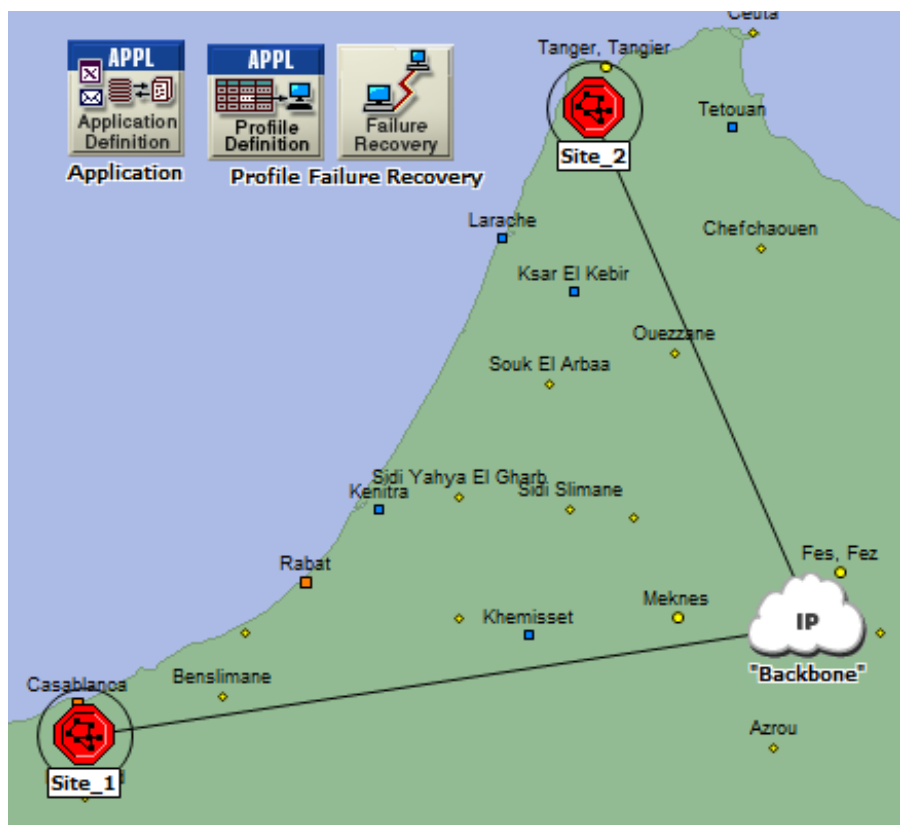

Fig. 2. Network topology

\section{B. Simulation parameters}

The proposed topology is based on the use of OSPF and EIGRP as routing protocol. All the possible combinations are compared and evaluated based on some quantitative metrics such as convergence duration, packet delay variation, end to end delay, jitter and throughput. These protocols are particularly chosen in order to get better performance for real time traffic such as video streaming and voice conferencing in the entire network.

In this section, a comparative analysis of EIGRP over OSPF is conducted. There are four network models, which are configured and ran as follows: first scenario with OSPF alone, second one with EIGRP alone, the third one with both, backbone OSPF and subnet EIGRP and forth one with both, backbone EIGRP and subnet OSPF concurrently. One failure link has been configured to occur at 300 seconds and to recover at 600 seconds.

The Table 1 below presents the various scenarios:

TABLE I. DIFFERENT COMBINATION OF EIGRP AND OSPF FOR DIFFERENT SCENARIOS

\begin{tabular}{|l|l|l|}
\hline Scenario name & Backbone & \multicolumn{1}{|c|}{ Sites } \\
\hline Back EIGRP_Sites OSPF & \multirow{2}{*}{ OSPF } & OSPF \\
Back OSPF_Sites EIGRP & & EIGRP \\
\hline EIGRP & \multirow{2}{*}{ EIGRP } & OSPF \\
\cline { 1 - 1 } OSPF & & EIGRP \\
\hline
\end{tabular}

For the traffic, we simulate voice traffic and use ftp application as background traffic, to evaluate the performance of a real time application, with the presence of another real traffic load configuration.

\section{Results}

Figures 3-8 present simulation results for different performance metrics: convergence time, end to end delay, delay variation, jitter, throughput, and packet loss rate.

From the Figure 3 below, it can be seen that the convergence time of EIGRP is faster than OSPF and EIGRP_OSPF combination. Because when the change occurs through the network, EIGRP detects the topology change and sends query to the immediate neighbours to have a successor and propagate this update to all routers. On the opposite side, the network convergence time of OSPF is slower than EIGRP and EIGRP_OSPF networks. As the change occurred in the OSPF network, all routers within an area update the topology database by flooding LSA to the neighbours and routing table is recalculated. Therefore, network convergence time of OSPF is getting slower than others.

As for EIGRP combined with OSPF, the convergence time is still important compared to EIGRP but slower than OSPF.

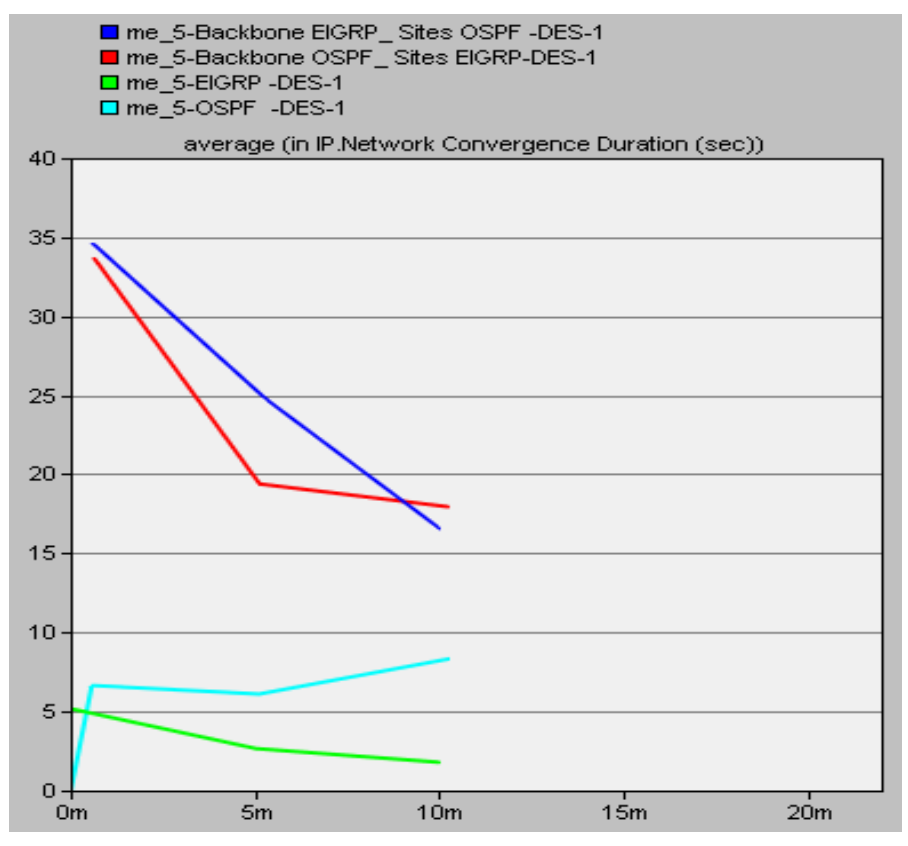

Fig. 3. Convergence time in seconds

End to end delay is defined as the time taken for a packet to be transmitted across a network from source to destination. Figure 4 shows that the use of one single routing protocol (EIGRP/OSPF) gives better results for end to end delay than having operators and sites configured with both routing protocols (redistribution). EIGRP still manifests better results. 


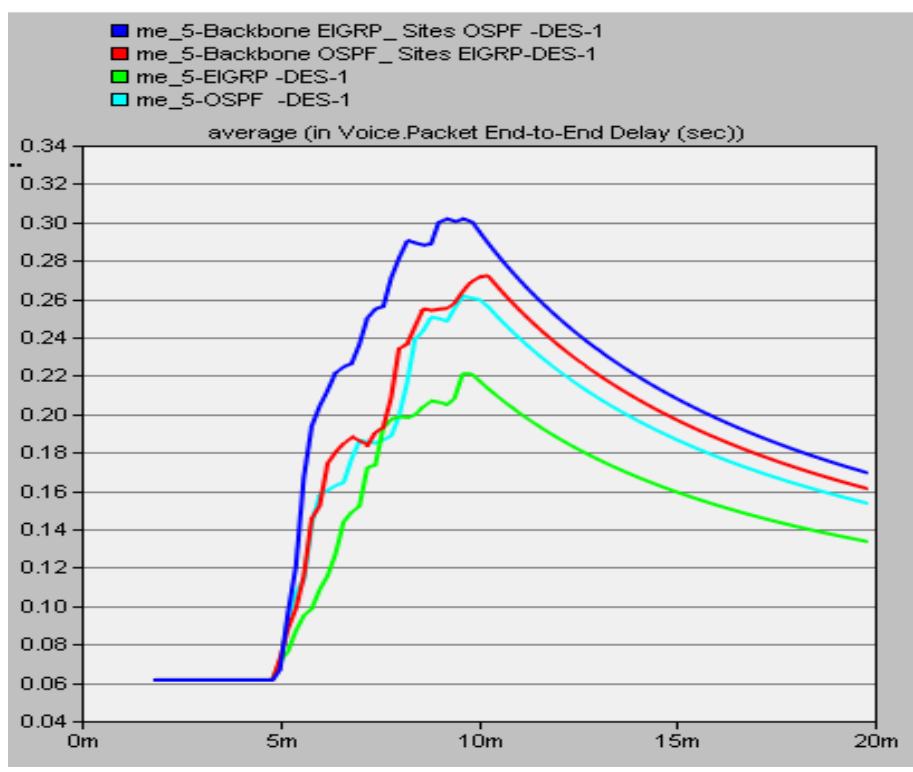

Fig. 4. End to End Delay in seconds

Packet delay variation is based on the difference in the end to end delay of selected packets; this metric has a significant impact on the quality of voice applications. From Figure 5, it can be noticed that delay variation of EIGRP network is smaller than delay variation observed for the three other networks. The highest delay variation is measured in the "Backbone EIGRP_Sites OSPF" network.

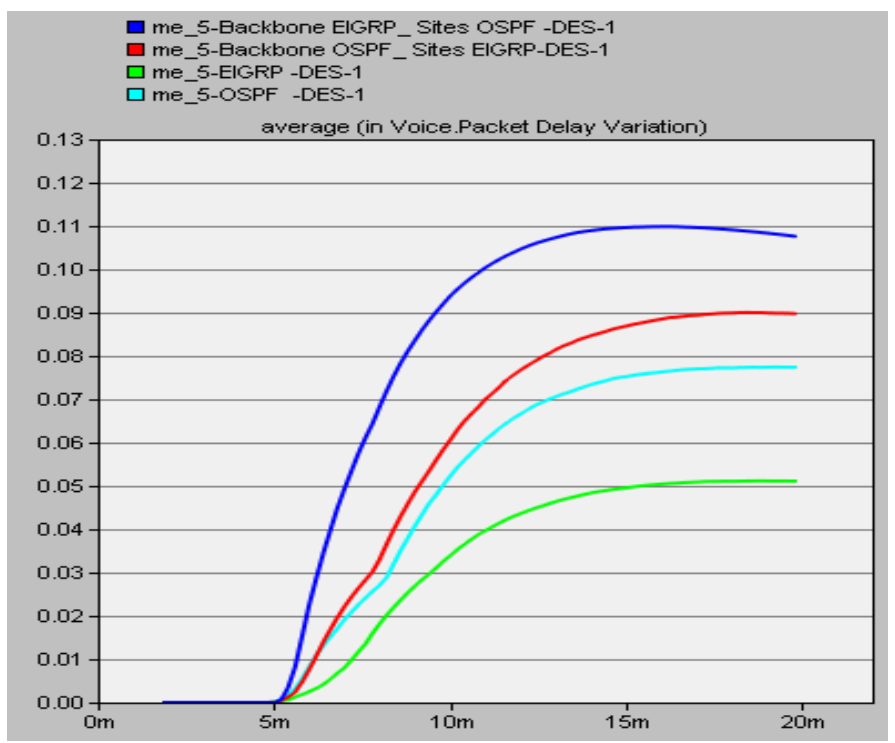

Fig. 5. Delay Variation

Jitter is simply the difference in packet delay; this factor should be as small as possible especially for voice application. Figure 6 presents the jitter metric for different scenarios. As shown, EIGRP has relatively the lowest jitter value in comparison with the three other scenarios, even compared with the case deploying redistribution.

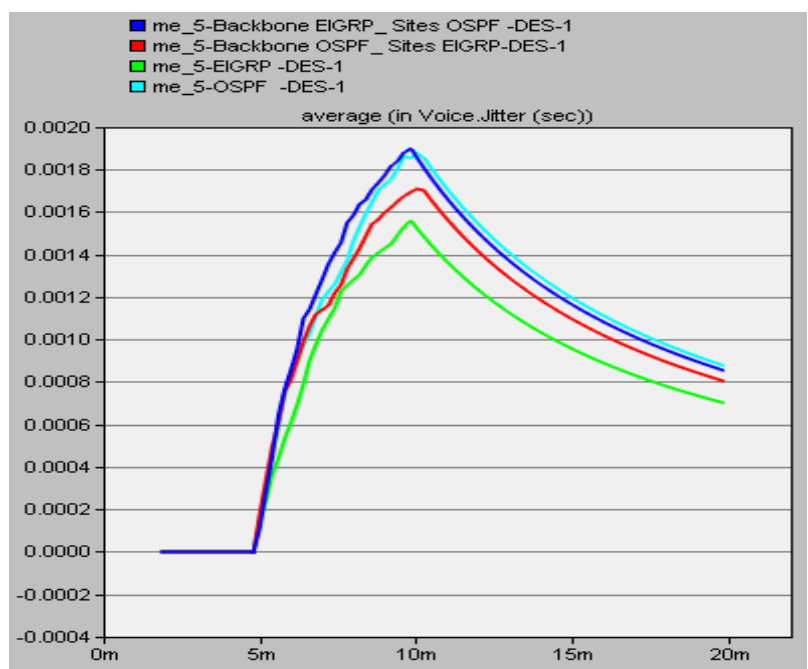

Fig. 6. Voice jitter

Throughput represents the average number of bits successfully received or transmitted by the receiver per unit time. Figure 7 indicates that before link failure, all scenarios give the same performance for the throughput metric. However, at the moment of the failure and before the link is recovered, using OSPF in the backbone or also in the company site gives better performance than the rest of protocol combinations.

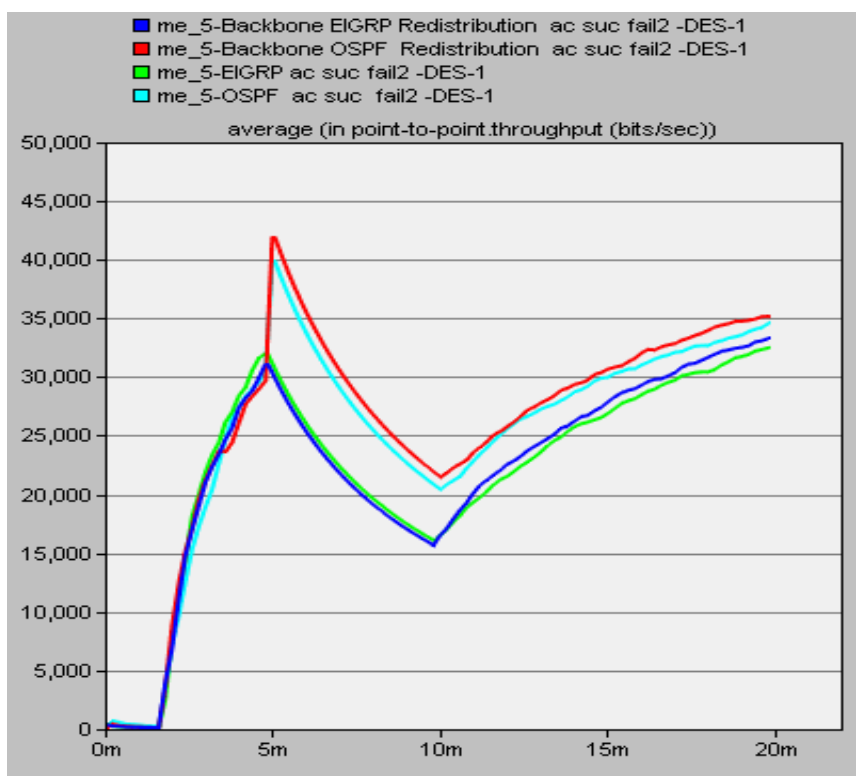

Fig. 7. Throughput

Packet loss occurs when one or more packets of data travelling across a network fail to reach their destination. Packet loss is typically caused by network congestion. It is measured as a percentage of packets lost with respect to packets sent. It is clear from the Figure 8 that the packet loss rate reaches the higher value in networks where there is redistribution. By comparing values of four scenarios, we can notice that the EIGRP scenario has the best performance represented by a small packet loss rate. 


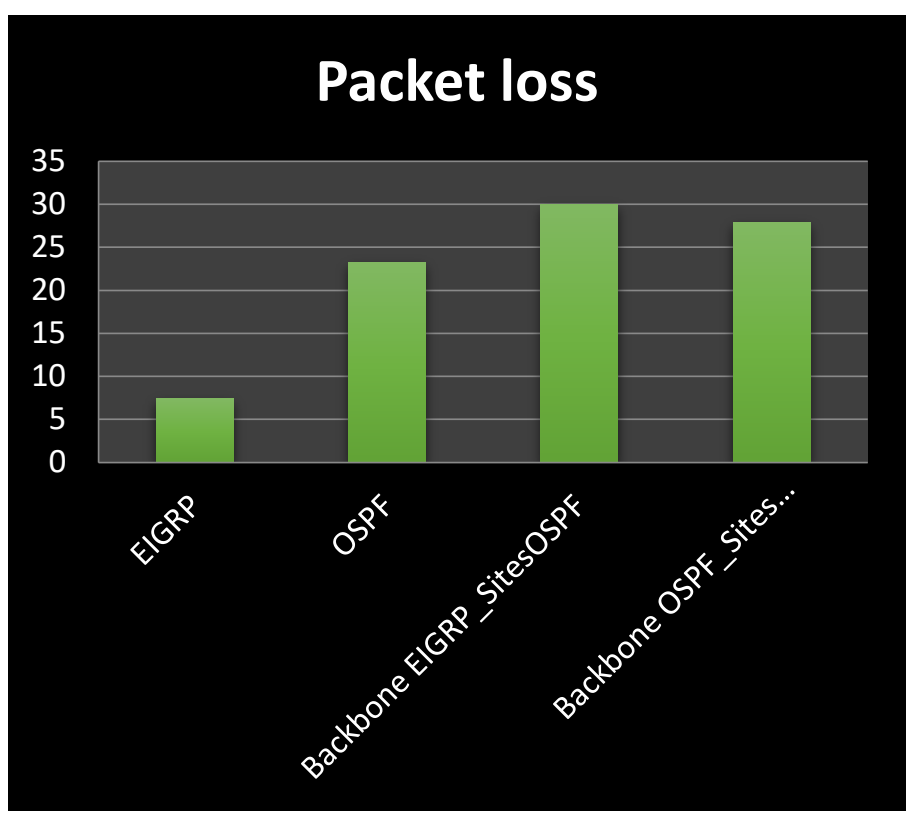

Fig. 8. Percentage of packet loss

\section{CONCLUSION AND PERSPECTIVES}

This study shows clearly that deploying one single routing protocol give always better results compared with the deployment of redistribution; however the schema or the scenarios are used and simulated in this deployment. This conclusion has already proved theoretically, and due to the loss of metric when transiting from EIGRP to OSPF and vice versa. Best performance is obtained while using EIGRP as a single routing protocol for all the network components.

In future work, a real experiment must be conducted to confirm simulation results and overpass the simulator limits.

\section{REFERENCES}

[1] Shewaye Sirika et Smita Mahajine, "Survey on Dynamic Routing Protocols », International Journal of Engineering Research \& Technology (IJERT), India, janv-2016.

[2] Mohsin Masood, Mohamed Abuhelala, et Ivan Glesk, « A comprehensive study of Routing Protocols Performance with Topological Changes in the Networks », International Journal of Advanced Information Science and Technology, Scotland, UK, 2016.
[3] M. Pavani, M. Sri Lakshmi, et Dr. S. Prem Kumar, «A Review on the Dynamic Routing Protocols in TCP/IP », The International Journal OF SCIENCE \& TECHNOLEDGE, India, mai-2014.

[4] Y.NavaneethKrishnan, Chandan N Bhagwat, et Aparajit Utpat, " Performance Analysis of OSPF and EIGRP Routing Protocols for Greener Internetworking »,2010.

[5] Amanpreet Kaur et Dinesh Kumar, « A SURVEY ON LINK STATE ROUTING PROTOCOLS », Bathinda.

[6] Archana C, «Analysis of RIPv2, OSPF, EI GRP Configuration on router Using CISCO Packet tracer», International Journal of Engineering Science and Innovative Technology (IJESIT) Volume 4, Issue 2, India, mars-2015.

[7] Vangala Rekha Andal, «Evaluation of EIGRP and OSPF Routing Protocols for Greener Internetworking », International Journal Of Emerging Trends In Technology And Sciences, Volume - 02, ISSUE 03, may-2014.

[8] Syed Yasir Jalali, Sufyan Wani, et Majid Derwesh, «Qualitative Analysis and Performance Evaluation of RIP, IGRP, OSPF and EGRP U sing OPNET TM », Advance in Electronic and Electric Engineering, Vol ume 4 , Number 4, India, apr-2014.

[9] N.Nazumudeen et C.Mahendran, «Performance Analysis o f Dynamic Routing Protocols Using Packet Tracer», International Journal of Innovative Research in Science, Engineering and Technology,Volume 3, Special Issue 1, févr-2014.

[10] Amrah Baba Ali, et Mujahid Tabassum, «A Comparative Study of IGP and EGP Routing Protocols, Performance Evaluation along Load Balancing and Redundancy across Different AS », Proceedings of the International MultiConference of Engineers and Computer Scientists 2016 Vol II, Hong Kong, mars-2016.

[11] Nisha Pandey et Dr. Dinesh Kumar, «Simulation Based Comparative Study on EIGRP/ IS-IS and OSPF/ IS-IS », International Journal of Engineering Research and General Science Volume 3, Issue 2, Part 2, India, avr-2015.

[12] S. Farhangi, A.Rostami, et S. Golmohammadi, «Performance Comparison of Mixed Protocols Based on EIGRP, IS-IS and OSPF for Real-time Applications », Middle-East Journal of Scientific Research, Iran, 2012.

[13] Emilija Stankoska, Nikola Rendevski, et Pece Mitrevski, «Simulation Based Comparative Performance Analysis of OSPF and EIGRP Routing Protocols », International Conference on Applied Internet and Information, Macedonia, 2016.

[14] Adarshpal S. Sethi et Vasil Y. Hnatyshin, The Practical OPNET User Guide for Computer Network Simulation.

[15] Jeffrey C. Mogul et Jean Tourrilhes, « DevoFlow: cost-effective flow management for high performance enterprise networks ", in Proceedings of the 9th ACM SIGCOMM Workshop on Hot Topics in Networks Article, Canada, 2010. 